\title{
Enhanced superconducting proximity effect in strongly correlated heterostructures
}

\author{
Chun Chen, ${ }^{1}$ Yan Chen, ${ }^{1,2}$ Z. D. Wang, ${ }^{2}$ and C. S. Ting ${ }^{3,1}$ \\ ${ }^{1}$ Department of Physics, State Key Laboratory of Surface Physics and Laboratory of Advanced Materials, \\ Fudan University, Shanghai, China \\ ${ }^{2}$ Department of Physics and Center of Theoretical and Computational Physics, The University of Hong Kong, \\ Pokfulam Road, Hong Kong, China \\ ${ }^{3}$ Texas Center for Superconductivity and Department of Physics, University of Houston, Houston, Texas 77204, USA
}

(Received 9 May 2010; published 3 November 2010)

\begin{abstract}
The electronic properties of a strongly correlated heterostructure consisting of $t$ - $J$ layer and metallic layer have been investigated by using the Gutzwiller projected mean-field approximation. Considering the proximity effect due to the large pseudogap energy scale of $t$ - $J$ layer, a large superconducting gap could be induced on the metallic layer. This enhanced superconducting gap may be even larger than that of the $t$ - $J$ layer. Related physical quantities including spectral functions and density of states are obtained. The consequences of these results on experiments are discussed.
\end{abstract}

DOI: 10.1103/PhysRevB.82.174502

PACS number(s): 74.78.Fk, 71.10.Fd, 74.20.Fg

\section{INTRODUCTION}

Despite of intensive investigations both experimentally and theoretically, there is still no consensus regarding to the nature and origin of the pseudogap state in underdoped cuprate superconductors. ${ }^{1}$ This exotic phase exists above $T_{c}$ and serves as the normal state of a superconductor across a broad range of hole densities in the underdoped regime. Since the energy scale of the pseudogap state is much higher than that of superconducting (SC) state, a question arises whether it is plausible to increase $T_{c}$ by constructing cuprate heterostructures, which combine the high pairing energy of underdoped layers with the large carrier density of metallic layers. There are some recent interesting experiments on heterostructures involving metallic-ion oxides. ${ }^{2-7}$ Such scenarios are inherently interesting due to the possibility that interface superconductivity can occur at temperatures above the maximum possible in bulk samples. In fact, experimental measurements on heterostructures have shown transition temperatures higher than that of either layer in isolation.

Theoretically, some proposals have been suggested to explain these phenomena. ${ }^{8-10}$ In particular, a simple twocomponent model consisted of a metallic layer and an underdoped pairing layer modeled by negative- $U$ Hubbard model, and suggested that $T_{c}$ of the whole system can be greatly enhanced by increasing the interlayer coupling. However this negative- $U$ model is over simplified and the doped Mott insulator physics has not been seriously considered in such composite systems. Moreover, there are some mean-field studies on the combinations of underdoped and overdoped layers. ${ }^{11,12}$ The SC order parameter in the overdoped layers is enhanced by the proximity effect of the strong pairing scale originating from the underdoped layers. The enhanced order parameter can even exceed the maximum value in uniform systems.

Although some microscopic theories have been proposed, the nature of the enhanced proximity effect has not been addressed in such details as to compare with or predict the experiments. In this paper, we study a composite system consisting of an underdoped cuprate layer modeled by a $t-J$
Hamiltonian and a metallic layer modeled by a tight-binding Hamiltonian. By employing the renormalized mean-field method, we investigate the pairing gap amplitudes of two individual layers at zero temperature. Due to the different correlation natures of these two layers, the interlayer coupling may lead to a much enhanced SC proximity effect and the SC order parameter on the metallic layer could be largely induced. Our results show that by increasing the interlayer coupling, the pairing gap of the $t$ - $J$ layer is suppressed while the SC gap on the metallic layer could be greatly enhanced. The maximal value of SC gap on metallic layer could be even greater than that of underdoped cuprate layer. Some physical quantities such as spectral function and the local density of states are calculated. The consequence of these results on experimental measurements will be discussed.

\section{FORMALISM}

The composite system we studied consists of a metallic layer as well as an underdoped $t$ - $J$ layer. As illustrated in Fig. 1, the model Hamiltonian can be described as

$$
H=H_{1}+H_{2}+H_{\perp},
$$

where $H_{1}$ is the Hamiltonian of the $t-J$ layer, which includes both nearest-neighbor and next-nearest-neighbor hopping terms on the square lattice,

$$
H_{1}=-t \sum_{\langle i, j\rangle, \sigma} P_{d}\left(c_{i, \sigma}^{\dagger} c_{j, \sigma}+\text { H.c. }\right) P_{d}+J \sum_{\langle i, j\rangle} \mathbf{S}_{i} \cdot \mathbf{S}_{j}-\mu \sum_{i} n_{1, i},
$$

where $c_{i, \sigma}^{\dagger}$ is an electron creation operator with spin $\sigma$ at site $i, S_{i}$ is a spin operator for electron, and $\mu$ is the chemical potential. The summations $\langle i, j\rangle$ run over all the nearestneighbor and second-nearest-neighbor links, and their corresponding hopping strengths are, respectively, $t$ and $t^{\prime} . P_{d}$ is a Gutzwiller projection operator to impose no double occupation of electrons at any site on the lattice. The second layer above the $t$ - $J$ layer is a metallic layer. Its Hamiltonian is given by 


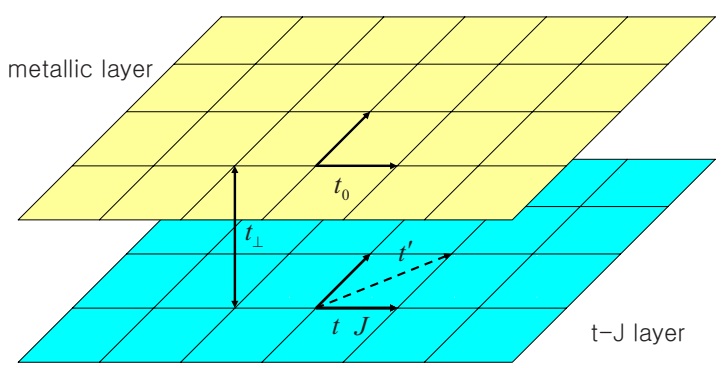

FIG. 1. (Color online) Schematic configuration of the composite bilayer system. The upper layer is metallic with hopping integral $t_{0}$ while the lower one is an underdoped $t$ - $J$ layer where nearestneighbor and next-nearest-neighbor hopping integrals are $t, t^{\prime}$, respectively, and the spin-spin coupling is $J$. The interlayer tunneling strength is $t_{\perp}$.

$$
H_{2}=-t_{0} \sum_{\langle i, j\rangle, \sigma} f_{i, \sigma}^{\dagger} f_{j, \sigma}+\text { H.c. }-\mu \sum_{i} n_{2, i},
$$

where $f_{i, \sigma}^{\dagger}$ denotes the electron creation operator of the second layer and $t_{0}$ stands for its hopping integral. Both layers share the same chemical potential $\mu$. The two layers are coupled by the single-particle interlayer tunneling Hamiltonian as

$$
H_{\perp}=-t_{\perp} \sum_{i, \sigma} P_{d} c_{i, \sigma}^{\dagger} P_{d} f_{i, \sigma}+\text { H.c. }
$$

where $t_{\perp}$ denotes the amplitude of interlayer tunneling. Hereafter we set $t=1, a=1$ and use $t$ as the energy unit and measure the length in units of lattice constant $a$, and choose $t_{0}$ $=0.5, t^{\prime}=-0.1$, and $J=0.3$.

Next we utilize the well-established Gutzwiller-projected wave-function technique and renormalized mean-field method; all the local constraints of no-double occupancy can be taken into account by an effective Hamiltonian ${ }^{13,14}$

$$
\begin{aligned}
H_{1}^{e f f}= & -g_{t} t \sum_{\langle i, j\rangle, \sigma} c_{i, \sigma}^{\dagger} c_{j, \sigma}+\text { H.c. }-g_{t} t^{\prime} \sum_{\langle i, j\rangle^{\prime}, \sigma} c_{i, \sigma}^{\dagger} c_{j, \sigma}+\text { H.c. } \\
& +g_{s} J \sum_{\langle i, j\rangle} \mathbf{S}_{i} \cdot \mathbf{S}_{j}-\mu \sum_{i} n_{1, i},
\end{aligned}
$$

where the two renormalization factors $g_{t}$ and $g_{s}$ are determined by the hole concentration $\delta_{1}$ of the $t$ - $J$ layer,

$$
g_{t}=\frac{2 \delta_{1}}{1+\delta_{1}}, \quad g_{s}=\frac{4}{\left(1+\delta_{1}\right)^{2}} .
$$

After decoupling the spin-spin superexchange interaction in $H_{1}$, we get the mean-field Hamiltonian of the composite system in a four-dimensional matrix form, ${ }^{15}$

$$
H_{e f f}=\sum_{\mathbf{k}} \psi_{\mathbf{k}}^{\dagger}\left(\begin{array}{cccc}
\zeta_{1, \mathbf{k}} & \tau & 0 & \varrho_{\mathbf{k}} \\
\tau & \zeta_{2, \mathbf{k}} & 0 & 0 \\
0 & 0 & -\zeta_{2, \mathbf{k}} & -\tau \\
\varrho_{\mathbf{k}} & 0 & -\tau & -\zeta_{1, \mathbf{k}}
\end{array}\right) \psi_{\mathbf{k}},
$$

where the four-component field operator using the Nambu formalism is $\psi_{\mathbf{k}}^{\dagger}=\left(c_{\mathbf{k}, \uparrow}^{\dagger} f_{\mathbf{k}, \uparrow}^{\dagger} f_{-\mathbf{k}, \downarrow} c_{-\mathbf{k}, \downarrow}\right) . \zeta_{1, \mathbf{k}}$ and $\zeta_{2, \mathbf{k}}$ correspond to the band dispersions of the $t-J$ and metallic layers, respectively. $\varrho_{1, \mathbf{k}}$ is from spin-spin exchange interaction term. Their explicit forms are given by

$$
\begin{gathered}
\zeta_{1, \mathbf{k}}=-2 g_{t} t\left(\cos k_{x}+\cos k_{y}\right)-4 g_{t} t^{\prime}\left(\cos k_{x} \cos k_{y}\right) \\
-\frac{3}{4} g_{s} J\left(\xi_{1, x} \cos k_{x}+\xi_{1, y} \cos k_{y}\right)-\mu, \\
\zeta_{2, \mathbf{k}}=-2 t_{0}\left(\cos k_{x}+\cos k_{y}\right)-\mu, \\
\varrho_{\mathbf{k}}=-\frac{3}{4} g_{s} J\left(\Delta_{1, x} \cos k_{x}+\Delta_{1, y} \cos k_{y}\right), \\
\tau=-\sqrt{g_{t}} t_{\perp},
\end{gathered}
$$

where the mean-field order parameters are

$$
\begin{aligned}
& \xi_{1, \hat{e}}=\left\langle c_{i, \uparrow}^{\dagger} c_{i+\hat{e}, \uparrow}+c_{i, \downarrow}^{\dagger} c_{i+\hat{e}, \downarrow}\right\rangle_{0}, \\
& \Delta_{1, \hat{e}}=\left\langle c_{i, \uparrow}^{\dagger} c_{i+\hat{e}, \downarrow}^{\dagger}-c_{i, \downarrow}^{\dagger} c_{i+\hat{e}, \uparrow}^{\dagger}\right\rangle_{0},
\end{aligned}
$$

with $\hat{e}= \pm x$ or $\pm y, i+\hat{e}$ denotes the nearest neighbor of site $i$ in the $\hat{e}$ direction, and the average of operators is performed in the unprojected BCS states. Here we focus on the translational-invariant state with the spin singlet and even parity SC pairing symmetry, where $\Delta_{1, i, i+\hat{e}}=\Delta_{1, i+\hat{e}, i}=\Delta_{1, \hat{e}}$ and $\xi_{1, i, i+\hat{e}}=\xi_{1, \hat{e}}$. The SC order parameter of $t$ - $J$ layer is related to mean-field pairing amplitude by $\Delta_{1, \hat{e}}^{\mathrm{SC}}=g_{t} \Delta_{1, \hat{e}}$. Antiferromagnetism is a possible order parameter in our studied system. In single-layer $t$ - $J$ model, antiferromagnetic order may exist at the very underdoped region (smaller than 0.03). The doping level in our present study is in the medium range. Here the appearance of antiferromagnetic state has not been considered for simplification.

The effective Hamiltonian $H_{\text {eff }}$ can be diagonalized by performing an unitary transformation as $\psi_{\mathbf{k}}^{i}=\sum_{j=1}^{4} v_{j}^{i} \phi_{\mathbf{k}}^{j}$, where the matrix element $v_{j}^{i}$ is $\mathbf{k}$ dependent and the quasiparticle field operator is $\phi_{\mathbf{k}}^{\dagger}=\left(d_{\mathbf{k}, \uparrow}^{\dagger} g_{\mathbf{k}, \uparrow}^{\dagger} g_{-\mathbf{k}, \downarrow} d_{-\mathbf{k}, \downarrow}\right)$. The four eigenvalues of $H_{e f f}$ can be obtained,

$$
E_{ \pm}(\mathbf{k})=\sqrt{w_{+}^{2}+\tau^{2} \pm \sqrt{w_{-}^{4}+\left(\varrho_{\mathbf{k}}^{2}+V_{\mathbf{k}}^{2}\right) \tau^{2}}},
$$

where $w_{ \pm}^{2}=\left(\zeta_{1, \mathbf{k}}^{2}+\varrho_{\mathbf{k}}^{2} \pm \zeta_{2, \mathbf{k}}^{2}\right) / 2, \quad V_{\mathbf{k}}=\zeta_{1, \mathbf{k}}+\zeta_{2, \mathbf{k}}$ and $E_{1}=E_{+}$, $E_{2}=E_{-}, E_{3}=-E_{-}, E_{4}=-E_{+}$. In the case of $\tau=0$, the quasiparticle spectra are reduced to decoupled $t-J$ model and tightbinding model. Because of the study of the SC proximity effect for the composite system, we need to define two extra mean-field order parameters $\xi_{2, \hat{e}}$ and $\Delta_{2, \hat{e}}=\Delta_{2, \hat{e}}^{\mathrm{SC}}$ on the metallic layer,

$$
\begin{aligned}
& \xi_{2, \hat{e}}=\left\langle f_{i, \uparrow}^{\dagger} f_{i+\hat{e}, \uparrow}+f_{i, \downarrow}^{\dagger} f_{i+\hat{e}, \downarrow}\right\rangle_{0}, \\
& \Delta_{2, \hat{e}}=\left\langle f_{i, \uparrow}^{\dagger} f_{i+\hat{e}, \downarrow}^{\dagger}-f_{i, \downarrow}^{\dagger} f_{i+\hat{e}, \uparrow}^{\dagger}\right\rangle_{0} .
\end{aligned}
$$

In terms of the new operators $d^{\dagger}, d$ and $g^{\dagger}, g$, these four mean fields can be expressed at zero temperature as

$$
\xi_{1, \hat{e}}=\frac{2}{N} \sum_{\mathbf{k}}\left(\left|v_{3}^{1}\right|^{2}+\left|v_{4}^{1}\right|^{2}\right) \cos k_{\hat{e}},
$$




$$
\begin{aligned}
& \Delta_{1, \hat{e}}=\frac{2}{N} \sum_{\mathbf{k}}\left(v_{3}^{1} v_{3}^{4}+v_{4}^{1} v_{4}^{4}\right) \cos k_{\hat{e}}, \\
& \xi_{2, \hat{e}}=\frac{2}{N} \sum_{\mathbf{k}}\left(\left|v_{3}^{2}\right|^{2}+\left|v_{4}^{2}\right|^{2}\right) \cos k_{\hat{e}}, \\
& \Delta_{2, \hat{e}}=\frac{2}{N} \sum_{\mathbf{k}}\left(v_{3}^{2} v_{3}^{3}+v_{4}^{2} v_{4}^{3}\right) \cos k_{\hat{e}},
\end{aligned}
$$

where $N$ denotes the number of sites on two-dimensional lattice. Together with definition of the hole concentrations on two layers $\delta_{1}=1-N_{1} / N, \delta_{2}=1-N_{2} / N+\left(N_{2} / 2 N\right)^{2}$, we can self-consistently determine the SC order parameters by numerically solving the above equations. Here $N_{1}$ and $N_{2}$ correspond to the electron numbers on the $t-J$ and metallic layers, respectively.

Next we need compute the physical quantities such as spectral function and density of states directly from the bare single-particle Green's function. Angle-resolved photoemission spectroscopy (ARPES) is one of the efficient techniques to probe the electronic structure. The ARPES result can be easily interpreted theoretically from a microscopic Hamiltonian approach because it measures the one-particle spectral function $A(k, \omega)$ with both momentum and energy information of the electrons inside solids. Thus we could analyze the physics of different regions in the momentum space at different energy scales. The bare single-particle Green's function in Nambu formalism is given by

$$
G_{0}(\mathbf{k}, t)=-i\left\langle T \psi_{\mathbf{k}}(t) \psi_{\mathbf{k}}^{\dagger}(0)\right\rangle
$$

and

$$
G_{0}(\mathbf{k}, i \omega)_{i j}=\sum_{n} \frac{v_{n}^{i} v_{n}^{j}}{i \omega-E_{n}(\mathbf{k})} .
$$

We then obtain the spectral function of the composite system as $A(\mathbf{k}, \omega)=-\frac{1}{\pi} \mathbf{I m}\left[G_{0}(\mathbf{k}, \omega+i \eta)_{11}+G_{0}(\mathbf{k}, \omega+i \eta)_{22}\right]$, where the first term represents the spectral function of $t-J$ layer and the second term denotes the spectral function of metallic layer. The Fermi surface can be determined according to the spectral weight at Fermi energy. The density of states is given by the imaginary part of the bare single-particle Green's function in real space. Scanning tunneling microscopy measures the spatially resolved local density of states (DOS) in terms of the differential conductance $d I / d V$ instead of the quantities in the momentum space.

\section{NUMERICAL RESULTS}

It is well known that the SC proximity effect is caused by the diffusion of electronic excitations in the superconductor. That is to say the single-particle tunneling interaction between two layers may induce the nonzero SC order parameter in the normal material. When we consider a heterostructure system consisting of a conventional BCS superconductor and a normal material, the enhancement of $T_{c}$ due to proximity effect is largest when the normal material is a metal with a large diffusivity rather than an insulator.
The critical temperature $T_{c}$ of the BCS superconductor is suppressed and indications of weak superconductivity are observed in the normal material. In our studied strongly correlated heterostructure, the underdoped cuprate layer has a high pairing energy scale while strong phase coherence of carriers exists in the metallic layer. According to the SC proximity effect, it is natural to predict that the metallic layer may have nonzero SC order parameter. Moreover, the main difference between the BCS superconductor and underdoped cuprate superconductor is the effect of strong correlations. In the composite system, the double occupation of electrons on each site is forbidden on the $t$ - $J$ layer while there is no such a constraint on metallic layer. When the single-particle interlayer tunneling interaction is switched on, an electron may hop onto the $t$ - $J$ layer only when the destination site is nonoccupied. This Mott physics effect is clearly shown in formula $(8 \mathrm{~d})$ where the interlayer tunneling strength is explicitly renormalized by a factor of $\sqrt{g_{t}}$ which is much larger than the renormalization factor $g_{t}$ on the $t-J$ layer. As shown in the following results, this effect may lead to the giant enhancement of superconductivity on the metallic layer.

To illustrate the effect of the strong correlation more transparently, we compare the SC order parameters $\Delta_{\mathrm{SC}}$ of these two layers at different values of $t_{\perp}$ as functions of doping levels $\delta_{1}$ and $\delta_{2}$. In the absence of interlayer tunneling, it is obvious that there is no SC order parameter on metallic layer and the doping dependence of SC order parameter on $t$ - $J$ layer follows a nonmonotonic domelike shape. ${ }^{13-16}$ This dome shape is a combinatorial effect of mean-field gap and Gutzwiller projection factor as a function of hole doping. In the presence of interlayer tunneling, electrons on the metallic layer may have an induced superconductivity due to the SC proximity effect which is indirectly generated by the spin-spin superexchange interaction on the $t$ - $J$ layer. As shown in Figs. 2(a) and 2(d), a small finite $t_{\perp}$ may suppress the amplitude of $\Delta_{\mathrm{SC}}$ on the $t$ - $J$ layer while the $\mathrm{SC}$ order parameter on metallic layer shows up due to proximity effect. With the increasing of $t_{\perp}$, we can observe that the SC order parameter on metallic layer can be greatly enhanced. When $t_{\perp}$ is close to 0.3 , the values of SC order parameters on two layers are comparable. When $t_{\perp}$ is further increased as depicted in Figs. 2(c) and 2(f), a large order parameter appears on the metallic layer and its value is much larger than that of $t-J$ layer which indicates a giant SC proximity effect due to strong correlation effect. From the conventional proximity effect, we know that the induction of superconductivity in normal materials may result in the suppression of the SC order parameter in original superconductors. This effect is clearly shown in Fig. 2. With the increasing of interlayer coupling, we observe the suppression of superconductivity on $t$ - $J$ layer while the SC order parameter increases on metallic layer. As illustrated in Fig. 2(c), the remarkable enhancement of SC order parameter on metallic layer may lead to the suppression of SC order parameter around $\delta_{1}=0.2$ region and the two-order-parameter peak structure appears.

In our theoretical treatment, these two coupled layers may mutually influence each other. For a small finite interlayer coupling strength, the SC order parameter as a function of doping level on each layer exhibits a single peak structure 

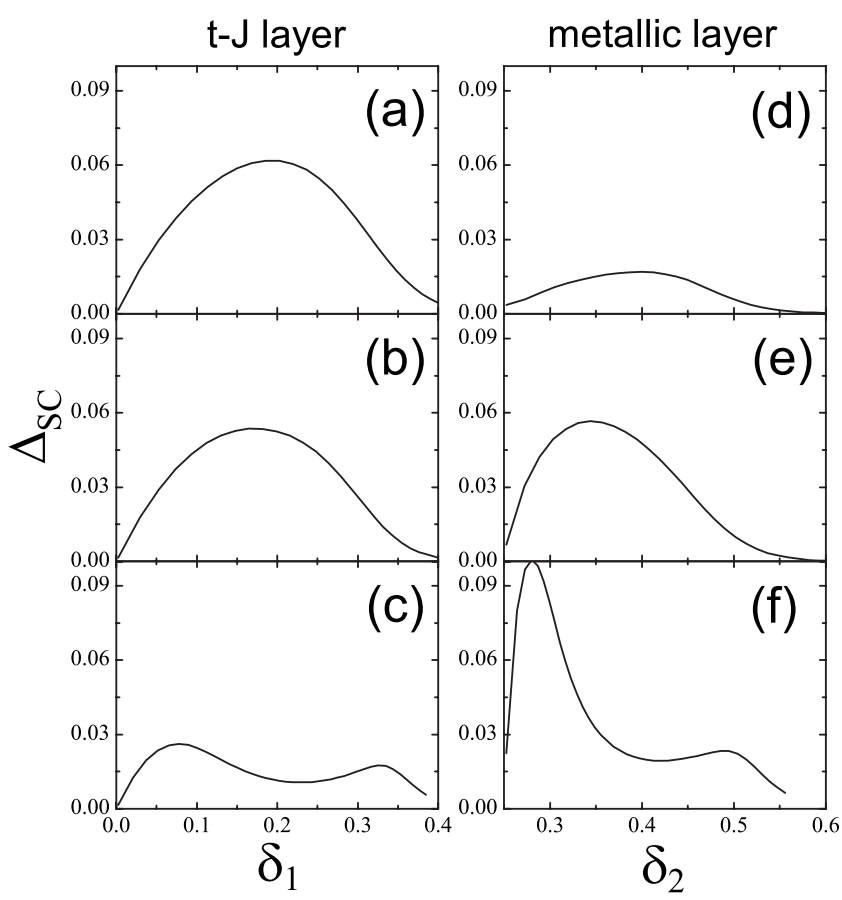

FIG. 2. Superconducting order parameters $\Delta_{1, \hat{e}}^{\mathrm{SC}}$ of the $t$ - $J$ layer (the left column) and $\Delta_{2, \hat{e}}^{\mathrm{SC}}$ of the metallic layer (the right column) with various interlayer hopping strengths $t_{\perp}$ [0.1 for (a) and (d), 0.3 for (b) and (e), and 0.8 for (c) and (f)] are plotted as functions of doping densities $\delta_{1}$ and $\delta_{2}$, respectively.

while two-peak structure shows up for quite strong $t_{\perp}$. As a matter of fact, the bandwidth of the metallic layer may affect the proximity effect and the wider metallic bandwidth may lead to the proximity effect weaker. Meanwhile we find out that the mean-field amplitude $\xi_{2, \hat{e}}$ will be greatly suppressed by increasing the doping level on metallic layer and the SC order parameter is remarkably enhanced. In other words, the suppression of kinetic energy in metallic layer may attribute to the enhancement of SC order parameter in metallic layer via the interlayer tunneling interaction. Note that the above qualitative behaviors do not depend on the choice of different Hamiltonian parameters.

To better compare with or predict the future ARPES experiments, we investigate the evolution of spectral function as well as Fermi surface as a function of energy. Before proceeding to the composite system, we work on the spectral function for a single $t$ - $J$ layer. Due to the nature of $d$-wave symmetry of mean-field pairing, the gap is vanishingly small in the nodal region around $(\pi / 2, \pi / 2)$ and reaches maximum near the antinodal region around $(\pi, 0)$ and $(0, \pi)$. So the spectral weight will be nonzero only around four nodal points while the spectral weight at other regions along the Fermi surface will be greatly suppressed by the finite pairing gap, as displayed in Fig. 3(a). Away from the Fermi energy, as shown in Figs. 3(b)-3(e), a sort of Fermi pocket structure shows up. Both the shapes, areas and orientations of the Fermi pockets depend on the energy deviations from the Fermi level and their signs. So by gradually increasing of the energy, the Fermi pocket will be elongated along the underlying Fermi surface. For positive $\omega$, the spectral function corresponds to that of the hole pocket, and for negative $\omega$, it
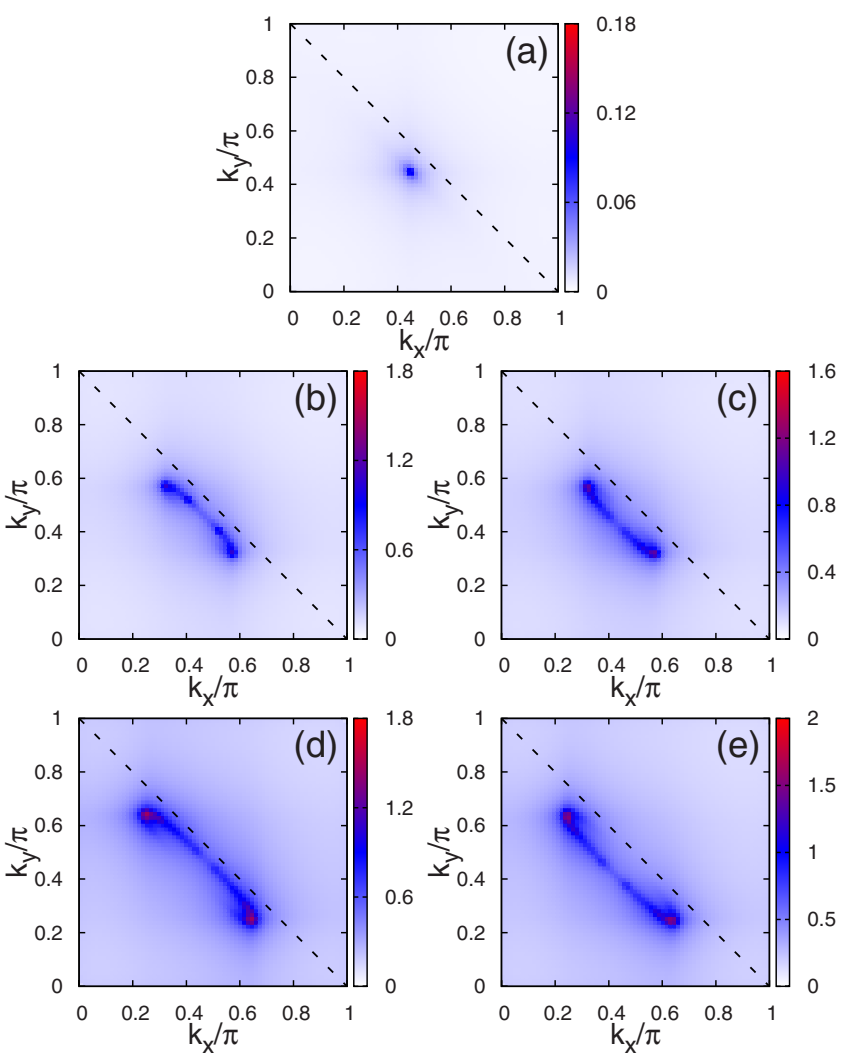

FIG. 3. (Color online) Evolution of spectral function $A(\mathbf{k}, \omega)$ of single $t$ - $J$ layer for various energies $\omega$. Panels (a)-(e) correspond to $\omega=0.0,0.1,-0.1,0.15$, and -0.15 , respectively. The chemical potential $\mu$ is fixed at -0.3 .

corresponds to the electron pocket. One side of the inner part of the Fermi pocket has much weaker spectral weight due to the contribution of coherence factor. The Fermi pocket structures at finite $\omega$ obtained in our calculation may be relevant to the observation of the ARPES experiments ${ }^{17}$ with a small energy window at the Fermi level.

Next we consider how the interlayer tunneling influences the spectral weight distribution of the composite system. In the absence of $t_{\perp}$, it is obvious that only the nodal point shows up on $t-J$ layer while the whole Fermi surface appears on the metallic layer. The introduction of interlayer tunneling interaction may give rise to two major physical effects: one is the single-particle band splitting, another is the SC proximity effect. Then the spectral weight distribution of the composite system might be dramatically reconstructed. In the left panels of Fig. 4, we show the evolution of spectral weight on $t$ - $J$ layer for different $t_{\perp}$. By increasing of interlayer tunneling strength, the original nodal point becomes more dispersed and finally evolves into two separated points [see Fig. 4(c)] because of the band splitting effect. In the right panels of Fig. 4, we show the evolution of Fermi surface on the metallic layer for various $t_{\perp}$. With the increasing of $t_{\perp}$, the spectral weights around the original Fermi surface are gradually suppressed. It is worth to mention that the suppression of the spectral weight becomes anisotropic in momentum space. As shown in Fig. 4(f), the proximity effect on metallic layer becomes more pronounced and consequently the large induced $d$-wave SC order parameter may strongly 

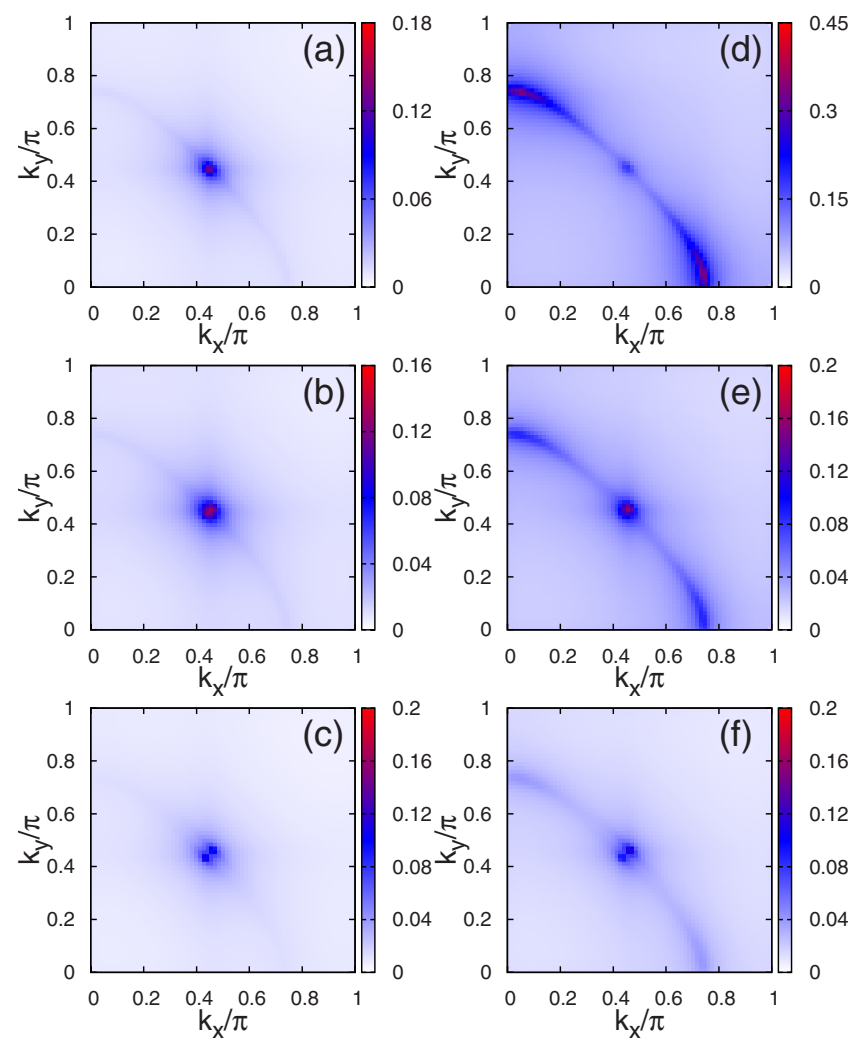

FIG. 4. (Color online) Evolution of spectral weight as a function of interlayer tunneling strength $t_{\perp}$ at Fermi energy. The left panels $[(\mathrm{a})-(\mathrm{c})]$ and right panels $[(\mathrm{d})-(\mathrm{f})]$ belong to the $t$ - $J$ layer and metallic layer, respectively. Panels [(a) and (d)], [(b) and (e)], and [(c) and (f) $]$ correspond to $t_{\perp}=0.05,0.1$, and 0.15 , respectively. The chemical potential $\mu$ is fixed at -0.3 .

suppress the spectral weight away from the nodal point.

Finally we study the DOS spectrum of the composite system. As expected, same physics of enhanced SC proximity effect will be shown in DOS spectrum as well. At the limit $t_{\perp}=0$, a $d$-wave gap structure is clearly shown on $t-J$ layer. This mean-field gap is pseudogap instead of SC gap. Once we switch on the interlayer tunneling interaction both the single-particle band splitting and the proximity effect may exhibit themselves, as illustrated in Fig. 5. The main features of DOS spectrum on one layer may exhibit its one-to-one correspondences of peak features and energy positions on the counterpart layer. In other words the DOS spectra on such two layers can be regarded like mirror images. As already displayed in Fig. 4, due to a finite interlayer coupling, the Fermi surfaces of two layers would be split. In real-space DOS spectrum, the original pseudogap coherent peak may be further split into two separated peaks and the separation distance between two peaks is determined by the strength of interlayer tunneling. In the left panels Figs. 5(a)-5(c) for $t-J$ layer, by increasing the interlayer tunneling strength, the pseudogap structure exhibits no much change while a new low-energy $d$-wavelike SC gap structure emerges and its gap value progressively increases. Meanwhile the right panels Figs. 5(d)-5(f) for metallic layer, a similar low-energy $d$-wavelike SC gap structure is clearly shown up and its coherence peak is remarkably high. The emerged $d$-wavelike
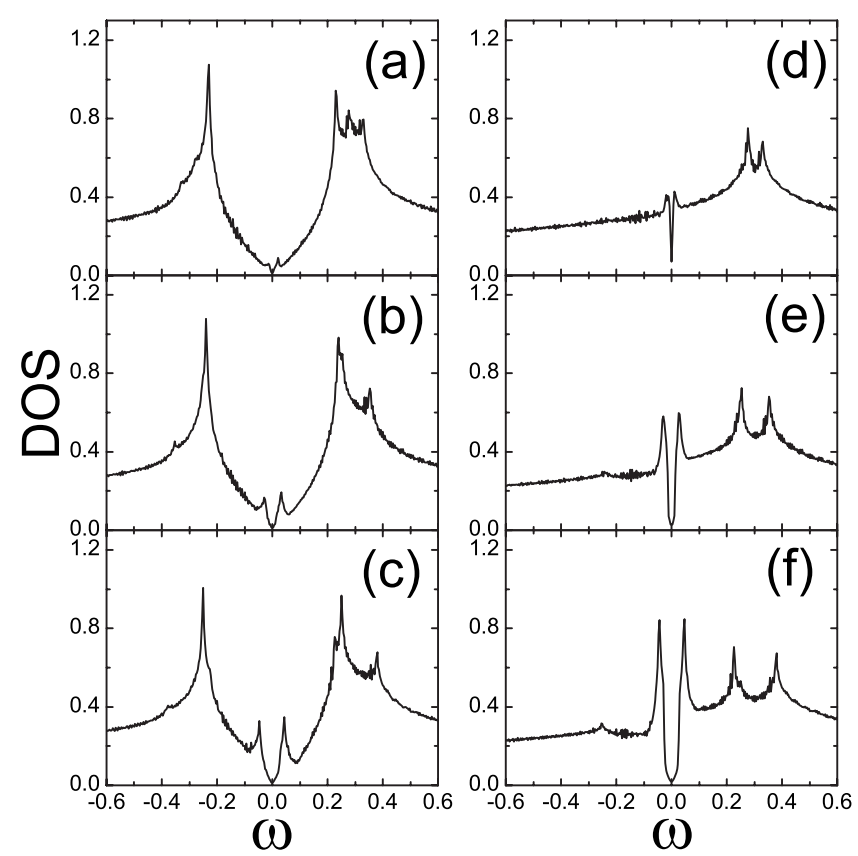

FIG. 5. Density of states in the $t-J$ layer $[(\mathrm{a})-(\mathrm{c})]$ and in the metallic layer $[(\mathrm{d})-(\mathrm{f})]$. Panels $[(\mathrm{a})$ and $(\mathrm{d})],[(\mathrm{b})$ and $(\mathrm{e})]$, and $[(\mathrm{c})$ and (f)] correspond to $t_{\perp}=0.05,0.1$, and 0.15 , respectively. The chemical potential $\mu$ is set to be -0.3 .

SC gap on metallic layer is induced by the large pseudogap energy scale of the $t-J$ layer due to the enhanced SC proximity effect. Besides we notice that the DOS peaks have more structures at positive energy region than negative energy region because the whole system is hole doped. It is worth to mention that the relationship between SC order parameter and SC gap is nontrivial in the composite system. For the weak interlayer coupling strength $t_{\perp}$, the pairing gap is essentially the pseudogap which is much larger than the $\mathrm{SC}$ gap on the $t-J$ layer. On the metallic layer, the SC gap is defined as half of the distance between the coherent peaks in Figs. 5(d)-5(f). It is not the SC order parameter as discussed in Figs. 2(d)-2(f).

Even though the present mean-field calculations apply only at zero temperature. We emphasize here that the strongly correlated heterostructures can really enhance the $\mathrm{SC}$ order parameter on metallic layer via the proximity effect. At finite temperatures, the SC metallic layer may have large phase stiffness. We expect a larger order parameter at zero temperature might give rise to higher $T_{c}$ at least in the approximation of mean-field level. Our theoretical results agree with the experimental observations qualitatively. In Ref. 3, the proximity effect in both $\mathrm{Au} / \mathrm{La}_{2-x} \mathrm{Sr}_{x} \mathrm{CuO}_{4}$ and $\mathrm{La}_{1.55} \mathrm{Sr}_{0.45} \mathrm{CuO}_{4} / \mathrm{La}_{2-x} \mathrm{Sr}_{x} \mathrm{CuO}_{4}$ bilayers has been investigated. Their experimental results provided a strong evidence of performed pairs in such bilayer systems. In particular, they found the proximity-induced gaps would persist over $T_{c}$ and up to nearly pseudogap temperature. Our theoretical results suggest that the enhanced superconducting proximity effect in strongly correlated heterostructures is driven by the pseudogap energy scale in the $t-J$ layer. That is to say, the appearance of proximity-induced gap may always show up below the pseudogap temperature. 


\section{SUMMARY}

We have studied the electronic properties of a strongly correlated heterostructure consisting of underdoped cuprate and metallic layers by using renormalized mean-field method. The large pseudogap energy scale of the $t-J$ layer may induce a large SC gap on the metallic layer due to the enhanced SC proximity effect. The induced SC gap could be even larger than that of the $t-J$ layer. We calculate the related physical quantities such as spectral function and density of state of the composite system. By analyzing these quantities as functions of interlayer coupling and energies, we obtain some interesting physical properties of strongly correlated heterostructures. Our results may shed light on the under- standing of the enhanced superconductivity in such structures.

\section{ACKNOWLEDGMENTS}

This work was supported by the National Natural Science Foundation of China (Grants No. 10874032 and No. 11074043) and the State Key Programs of China (Grant No. 2009CB929204), Shanghai Municipal Government, the RGC grants in HKSAR, and the Robert A. Welch Foundation under Grant No. E-1146 and the Texas Center for Superconductivity at the University of Houston through the State of Texas.
${ }^{1}$ T. Timusk and B. Statt, Rep. Prog. Phys. 62, 61 (1999).

${ }^{2}$ O. Yuli, I. Asulin, O. Millo, D. Orgad, L. Iomin, and G. Koren, Phys. Rev. Lett. 101, 057005 (2008).

${ }^{3}$ O. Yuli, I. Asulin, Y. Kalcheim, G. Koren, and O. Millo, Phys. Rev. Lett. 103, 197003 (2009).

${ }^{4}$ G. Koren and O. Millo, Phys. Rev. B 80, 054507 (2009).

${ }^{5}$ A. Gozar, G. Logvenov, L. F. Kourkoutis, A. T. Bollinger, L. A. Giannuzzi, D. A. Muller, and I. Bozovic, Nature (London) 455, 782 (2008).

${ }^{6}$ G. Logvenov, A. Gozar, and I. Bozovic, Science 326, 699 (2009).

${ }^{7}$ C. V. Parker, A. Pushp, A. N. Pasupathy, K. K. Gomes, J. S. Wen, Z. J. Xu, S. Ono, G. D. Gu, and A. Yazdani, Phys. Rev. Lett. 104, 117001 (2010).

${ }^{8}$ S. A. Kivelson, Physica B 318, 61 (2002).

${ }^{9}$ E. Berg, D. Orgad, and S. A. Kivelson, Phys. Rev. B 78, 094509 (2008).
${ }^{10}$ D. H. Lee, Phys. 1, 19 (2008).

${ }^{11}$ S. Okamoto and T. A. Maier, Phys. Rev. Lett. 101, 156401 (2008).

${ }^{12}$ L. Goren and E. Altman, Phys. Rev. B 79, 174509 (2009).

${ }^{13}$ F. C. Zhang, C. Gros, T. M. Rice, and H. Shiba, Supercond. Sci. Technol. 1, 36 (1988).

${ }^{14}$ P. W. Anderson, P. A. Lee, M. Randeria, T. M. Rice, N. Trivedi, and F. C. Zhang, J. Phys.: Condens. Matter 16, R755 (2004).

${ }^{15}$ H. X. Huang, Y. Q. Li, J. Y. Gan, Y. Chen, and F. C. Zhang, Phys. Rev. B 75, 184523 (2007).

${ }^{16}$ M. Ogata and H. Fukuyama, Rep. Prog. Phys. 71, 036501 (2008).

${ }^{17}$ J. Q. Meng, G. D. Liu, W. T. Zhang, L. Zhao, H. Y. Liu, X. W. Jia, D. X. Mu, S. Y. Liu, X. L. Dong, W. Lu, G. L. Wang, Y. Zhou, Y. Zhu, X. Y. Wang, Z. Y. Xu, C. T. Chen, and X. J. Zhou, Nature (London) 462, 335 (2009). 